TRANSACTIONS OF THE

AMERICAN MATHEMATICAL SOCIETY

Volume 355, Number 12, Pages 4985-5002

S 0002-9947(03)03223-9

Article electronically published on July 24, 2003

\title{
A PAIR OF DIFFERENCE DIFFERENTIAL EQUATIONS OF EULER-CAUCHY TYPE
}

\author{
DAVID M. BRADLEY
}

\begin{abstract}
We study two classes of linear difference differential equations analogous to Euler-Cauchy ordinary differential equations, but in which multiple arguments are shifted forward or backward by fixed amounts. Special cases of these equations have arisen in diverse branches of number theory and combinatorics. They are also of use in linear control theory. Here, we study these equations in a general setting. Building on previous work going back to de Bruijn, we show how adjoint equations arise naturally in the problem of uniqueness of solutions. Exploiting the adjoint relationship in a new way leads to a significant strengthening of previous uniqueness results. Specifically, we prove here that the general Euler-Cauchy difference differential equation with advanced arguments has a unique solution (up to a multiplicative constant) in the class of functions bounded by an exponential function on the positive real line. For the closely related class of equations with retarded arguments, we focus on a corresponding class of solutions, locating and classifying the points of discontinuity. We also provide an explicit asymptotic expansion at infinity.
\end{abstract}

\section{INTRODUCTION}

We study two classes of linear difference differential equations analogous to EulerCauchy ordinary differential equations, but in which multiple arguments are shifted forward or backward by fixed amounts. Special cases of these equations have been studied quite extensively in the context of certain problems in number theory and combinatorics - see $\sqrt{2}$ for a brief overview. Here, we take a broader viewpoint. As in [9], we focus primarily on the advanced-argument linear difference differential equation

$$
u q^{\prime}(u)=\sum_{j=0}^{m} \alpha_{j} q\left(u+v_{j}\right), \quad u>0
$$

in which the argument $u$ is incremented by the non-negative real numbers $0=v_{0}<$ $v_{1}<\cdots<v_{m}$, and the coefficients $\alpha_{0}, \alpha_{1}, \ldots, \alpha_{m}$ are arbitrary complex numbers. Here, however, we study (1.1) in conjunction with the related delay differential

Received by the editors March 19, 2002 and, in revised form, October 15, 2002.

2000 Mathematics Subject Classification. Primary 34K06; Secondary 34K12, 34K25.

Key words and phrases. Difference differential equations, integral transforms, adjoint relation, Dickman-de Bruijn function, sieves.

This research was supported by the University of Maine summer faculty research fund. 
equation

$$
(u p(u))^{\prime}=-\sum_{j=0}^{m} \alpha_{j} p\left(u-v_{j}\right) .
$$

It turns out that by judiciously pairing equations from each class, one can infer properties of solutions to one equation from properties of a solution to the other. We exploit this relationship to significantly strengthen previous uniqueness results concerning equation (1.1). Our main uniqueness result is that (1.1) has a unique solution (up to a multiplicative constant) in the class of exponentially bounded functions.

To exploit the relationship between the two equations (1.1) and (1.2), we need to amass a certain amount of information concerning a solution to the latter. In this direction we establish new results, including the nature and location of discontinuities, and an explicit asymptotic expansion.

The organization of the paper is as follows. In \$2 a brief historical overview is provided. We discuss uniqueness of solutions to (1.1) in the class of polynomially bounded functions in $\$ 3$. The next section focuses on a special case of (1.1) arising in sieve theory and the study of cycle lengths of a random permutation. The particular properties of the parameters in this case are exploited to show that any solution to the underlying equation which is not polynomially bounded must be very wild indeed. The techniques of $\$ 4$ prepare the ground for our exponential uniqueness theorem. We motivate the introduction of the adjoint equation in \$5. Information concerning the asymptotic behavior of solutions to the adjoint equation is needed, and the relevant results are presented in $\$[6$ Details of the proof of our exponential uniqueness theorem are relegated to the penultimate section.

Notation. The Bachmann-Landau O-notation and the standard notion of asymptotic equivalence are briefly recalled in the forms that we wish to use them. If $g$ is a positive function of a positive real variable, the symbol $\mathrm{O}(g(u)), 0<u \rightarrow \infty$, denotes an unspecified function $f$ for which there exist positive real numbers $u_{0}$ and $B$ such that $|f(u)| \leq B g(u)$ for all real $u>u_{0}$. Two functions $f$ and $g$ are asymptotically equivalent at infinity, written $f(u) \sim g(u), 0<u \rightarrow \infty$, if for every $\varepsilon>0$, there exists a positive real number $u_{0}$ such that $|f(u)-g(u)|<\varepsilon|g(u)|$ whenever $u>u_{0}$.

\section{HistoricAl OVERVIEW}

Ever since Dickman [24] showed that the asymptotic density of the positive integers $n \leq x$ having no prime factors exceeding $x^{1 / u}$ is given by the continuous solution $\rho(\bar{u})$ to the difference differential equation

$$
u \rho^{\prime}(u)=-\rho(u-1), \quad u>1,
$$

with boundary condition

$$
\rho(u)=1, \quad 0<u \leq 1,
$$

it has been known that difference differential equations arise in the study of certain problems in analytic number theory. We now know of at least four types of number theoretical problems in which difference differential equations arise. These may be loosely classified according to the context of the original problem. The first type, "psixyology" [37], encompasses those problems associated with determining 
the probability distribution of the prime factors of an integer $6,11,12,14,24,30$. The second type, "sieve theory" [28], is concerned with the problem of estimating the size of a finite set of integers after certain residue classes have been eliminated [4. 10, 15, 16, 17, 18, 19, 20, 27, 33. The third type deals with the problems relating to the determination of the cycle decomposition of a random permutation $2,5,25$. 26 34, 38, 40, and is closely related to the first [1]. The fourth type focuses on estimating incomplete sums of multiplicative functions $33,13,29$.

Despite the extensive literature on the subject, apart from the treatment of a few isolated cases [7, 8, 21, 22, 23, 31, 32, 39, 41, 42. the main focus has been on the application to problems in number theory and combinatorics as opposed to the study of the underlying difference differential equations as a problem in its own right. Although a systematic study of equation (1.1) in full generality was initiated in [9], there is still additional work that remains to be done with regard to providing a comprehensive treatment. The present paper can be viewed as a further contribution in this respect, in that we explore more deeply the question of uniqueness, and reveal more fully the role of the adjoint equation (1.2). It is hoped that some of the analytical techniques used herein - and more generally our ongoing program of study - will be of interest to researchers in number theory and the wider differential equations community as well.

\section{Polynomially Bounded solutions}

As in 9], we fix a non-negative integer $m$, real numbers $0=v_{0}<v_{1}<\cdots<v_{m}$, complex numbers $\alpha_{0}, \alpha_{1}, \ldots, \alpha_{m}$, and put $\beta:=\alpha_{0}+\alpha_{1}+\cdots+\alpha_{m}$. Without loss of generality, we assume $\alpha_{j} \neq 0$ for $1 \leq j \leq m$. Define a function $q^{*}$ on the positive real half-line $(0 \ldots \infty)$ as follows.

Definition 1. For $u>0$, let

$$
q^{*}(u):=\frac{1}{\Gamma(-\beta)} \int_{0}^{\infty} x^{-\beta-1} \exp \left\{-u x+\sum_{j=1}^{m} \alpha_{j} \int_{0}^{x} \frac{1-e^{-v_{j} t}}{t} d t\right\} d x
$$

if $\beta$ is a negative integer, and

$$
q^{*}(u):=\frac{\Gamma(\beta+1)}{2 \pi i} \underset{\leftrightharpoons}{\leftrightharpoons} z^{-\beta-1} \exp \left\{u z-\sum_{j=1}^{m} \alpha_{j} \int_{0}^{z} \frac{e^{v_{j} t}-1}{t} d t\right\} d z
$$

otherwise.

The contour in (3.1b) starts at $-\infty$, hugs the lower side of the negative real axis, then circles the origin in the positive (counter-clockwise) direction before returning to $-\infty$ along the upper side of the negative real axis. By deforming the contour, one can readily check that (3.1a) and (3.1b) are equivalent if $\Re(\beta)<0$ and $\beta$ is not a negative integer. It follows [9, Cor. 2.5] that if $\alpha_{j}$ is real for each $j=0,1, \ldots, m$ and $\beta<0$, then $q^{*}$ is positive, convex, and log-convex. If $\beta$ is a non-negative integer, then we can close the contour and deform it into the unit circle, so that $q^{*}$ is then a polynomial of degree $\beta$. Otherwise, the singularity of the integrand is a branch point at $z=0$, and $q^{*}$ is a transcendental function. A detailed treatment of the various properties of $q^{*}$, including asymptotic expansions, behavior at 0 , and a representation of $q^{*}(u)$ as an exponential of a Hellinger type integro-differential operator acting on the monomial $u^{\beta}$, is given in [9]. 
The main interest in $q^{*}$ stems from the fact that it provides a $C^{\infty}$ solution to the difference differential equation (1.1). More specifically, combining Proposition 2.2 and Corollary 2.4 of [9] yields the following result.

Proposition 1. The function $q^{*}$ of Definition 1 satisfies the difference differential equation (1.1) and the asymptotic formula

$$
q^{*}(u) \sim u^{\beta}, \quad 0<u \rightarrow \infty .
$$

We recall [9, Theorem 3] that there is at most one function $q$ satisfying the difference differential equation (1.1) and the additional condition that $q(u)$ is asymptotic to a fixed power of $u$ for large real values of the argument $u$. Combining this result with Proposition 1 yields the following uniqueness result, which we record for future use:

Theorem 2. Suppose that $q$ satisfies the difference differential equation (1.1) and the additional requirement that there exists a complex number $\tau$ such that

$$
q(u) \sim u^{\tau}, \quad 0<u \rightarrow \infty .
$$

Then $\tau=\beta$ and $q=q^{*}$, where $q^{*}$ is as in Definition 1 .

In other words, the function $q^{*}$ of Definition 1 is the unique solution to the difference differential equation (1.1) in the class of functions asymptotic to a monomial at infinity. Theorem 4 of [9] strengthens Theorem[2] by widening the class of admissible functions to those functions which are polynomially bounded (i.e. majorized by a polynomial) in the right half-plane. The proof in [9] of this latter result is unnecessarily complicated, applying residue theory to the inverse Laplace transform of a suitably high order derivative of a supposed solution. Before proceeding further, it may be of interest to give a simpler proof which has the additional advantage of requiring only that the supposed solution be majorized by a polynomial on the positive real half-line.

Theorem 3. Suppose that $q$ satisfies the difference differential equation (1.1). Suppose further that there exists a real number $r$ such that

$$
q(u)=\mathrm{O}\left(u^{r}\right), \quad 0<u \rightarrow \infty .
$$

Then there exists a complex number $A$ such that for all $u>0, q(u)=A q^{*}(u)$, where $q^{*}$ is given by Definition 1. The multiplicative constant A may of course depend on the parameters $\alpha_{j}, v_{j}(j=0,1,2, \ldots, m)$ but not on $u$.

Proof. For any complex number $A$, Theorem 2 implies that $q=A q^{*}$ is the unique function satisfying both the difference differential equation (1.1) and the asymptotic formula

$$
q(u) \sim A u^{\beta}, \quad 0<u \rightarrow \infty .
$$

Accordingly, it is enough to show that our hypotheses imply that the asymptotic formula (3.4) holds for some complex number $A$, or equivalently, that

$$
\lim _{u \rightarrow \infty} u^{-\beta} q(u)
$$

exists.

We can write the difference differential equation (1.1) in the form

$$
\left(u^{-\beta} q(u)\right)^{\prime}=u^{-\beta-1} \sum_{j=1}^{m} \alpha_{j}\left\{q\left(u+v_{j}\right)-q(u)\right\}, \quad u>0 .
$$


We have dropped the term in the sum that corresponds to $j=0$. It vanishes because $v_{0}=0$. It follows that for $u>0$,

$$
\begin{aligned}
u^{-\beta} q(u) & =q(1)+\int_{1}^{u} t^{-\beta-1} \sum_{j=1}^{m} \alpha_{j}\left\{q\left(t+v_{j}\right)-q(t)\right\} d t \\
& =q(1)+\sum_{j=1}^{m} \alpha_{j} \int_{1}^{u} t^{-\beta-1} \int_{0}^{v_{j}} q^{\prime}(x+t) d x d t .
\end{aligned}
$$

Let $c:=\Re(\beta)$, and suppose that the polynomial growth requirement (3.3) holds. Since increasing $r$ only weakens the hypothesis, there is no harm in assuming that $r=n+c+1 / 2$ for some non-negative integer $n$. The stipulation that $r-c$ lies halfway between two consecutive integers is not strictly necessary; however, as it precludes the possibility that an integer power of $u$ multiplied by $u^{r-c}$ will integrate to a logarithm as opposed to a power of $u$, the number of cases to be considered in our subsequent estimates is conveniently reduced.

By the difference differential equation (1.1) and our hypothesis, we have

$$
\left|u q^{\prime}(u)\right| \leq \sum_{j=0}^{m}\left|\alpha_{j}\right|\left|q\left(u+v_{j}\right)\right|=\mathrm{O}\left(u^{r}\right), \quad 0<u \rightarrow \infty,
$$

and hence $q^{\prime}(u)=\mathrm{O}\left(u^{r-1}\right)$. Using this bound in the integral (3.5), we conclude that

$$
u^{-\beta} q(u)=q(1)+\mathrm{O}\left(\int_{1}^{u} t^{-c-1} t^{r-1} d t\right)=\mathrm{O}(1)+\mathrm{O}\left(u^{r-c-1}\right),
$$

and hence $q(u)=\mathrm{O}\left(u^{c}\right)+\mathrm{O}\left(u^{r-1}\right)$. By repeating the previous steps if necessary, we arrive at the growth estimate

$$
q(u)=\mathrm{O}\left(u^{c}\right), \quad 0<u \rightarrow \infty .
$$

Using this latter bound in the difference differential equation (1.1) yields

$$
\left|u q^{\prime}(u)\right| \leq \sum_{j=0}^{m}\left|\alpha_{j}\right|\left|q\left(u+v_{j}\right)\right|=\mathrm{O}\left(u^{c}\right), \quad 0<u \rightarrow \infty,
$$

and hence $q^{\prime}(u)=\mathrm{O}\left(u^{c-1}\right)$. It follows that the integrals

$$
\int_{1}^{\infty} t^{-\beta-1} \int_{0}^{v_{j}} q^{\prime}(x+t) d x d t \quad(j=0,1,2, \ldots, m)
$$

all converge. Therefore, we may let $u \rightarrow+\infty$ in (3.5), from which we infer that the limit

$$
A:=\lim _{u \rightarrow \infty} u^{-\beta} q(u)=q(1)+\sum_{j=1}^{m} \alpha_{j} \int_{1}^{\infty} t^{-\beta-1} \int_{0}^{v_{j}} q^{\prime}(x+t) d x d t
$$

exists. In other words,

$$
q(u) \sim A u^{\beta}, \quad 0<u \rightarrow \infty,
$$

and by our initial remarks, the proof is complete.

In 97. we show that the conclusion of Theorem 3 holds with (3.3) replaced by the weaker hypothesis that $q$ be majorized by some fixed power of an exponential function. Before proving this stronger result in full generality, it is instructive to examine an important special case which is used in the proof of the general case. 


\section{The equation $(u q(u))^{\prime}=\kappa q(u)-\kappa q(u+1)$}

Let $\kappa$ be a positive real number. The difference differential equation

$$
(u q(u))^{\prime}=\kappa q(u)-\kappa q(u+1), \quad u>0,
$$

was introduced by Iwaniec [33] as an adjoint equation for the upper and lower bounding sieve functions of Rosser's sieve, and is a special case of (1.1) with $m=1$, $v_{1}=1, \alpha_{0}=\kappa-1, \alpha_{1}=-\kappa$ and $\beta=\alpha_{0}+\alpha_{1}=-1$. The role of $\kappa$ in sieve theory is to measure the average number of residue classes being deleted for each prime used in the sifting. Equation (4.1) and its close cousin with the minus sign replaced by a plus sign have been studied fairly extensively in the context of sieve theory [7, 8, 17] 18, 19, 20, 21, 22, 33]. The case $\kappa=1$ of (4.1) also occurs in the problem of determining the asymptotic average size of the largest prime factor of a random integer and the longest cycle of a random permutation [25, 26, 34, 40]. More specifically, the average cycle length of the longest cycle in a permutation on $n$ symbols [40] and the average number of digits in the largest prime factor of an $n$-digit number [34] are both asymptotic to $n q_{1}^{\prime}(1) / q_{1}(1)$, where

$$
q_{1}(u):=\int_{0}^{\infty} \exp \left\{-u x-\int_{0}^{x} \frac{1-e^{-t}}{t} d t\right\} d x, \quad u>0,
$$

satisfies (4.1) with $\kappa=1$. We also have [41, 42 ]

$$
\#\left\{n \in \mathbf{Z}: 1 \leq n \leq x, P_{2}(n) \leq P_{1}(n)^{1 / u}\right\}=x e^{\gamma} q_{1}(u)+\mathrm{O}\left(\frac{x}{\log x}\right), \quad x \rightarrow \infty,
$$

where $P_{j}(n)$ denotes the $j$ th largest prime factor of $n$ and $\gamma$ is Euler's constant.

By the results of $\sqrt[3]{3}$, we know that the polynomially bounded solutions to (4.1) are all constant multiples of $q^{*}$, where here

$$
q^{*}(u)=\int_{0}^{\infty} \exp \left\{-u x-\kappa \int_{0}^{x} \frac{1-e^{-t}}{t} d t\right\} d x, \quad u>0
$$

It turns out that any solution of (4.1) which is not a constant multiple of (4.2) must oscillate wildly. More precisely, we show that if $q$ satisfies (4.1) with $\kappa>0$, and if there is no constant $A$ for which $q=A q^{*}$, then $q(u) \neq \mathrm{O}(\exp (\lambda u))$ for any fixed $\lambda>0$, and for all $u>0, q$ changes sign infinitely often in $[u, \infty)$. To prove this, we first establish the following special case of our main uniqueness result.

Lemma 4. Suppose that $q$ satisfies the difference differential equation (1.1) with $\beta=-1$. Suppose further that there exists a positive real number $\lambda$ such that

$$
q(u)=\mathrm{O}\left(e^{\lambda u}\right), \quad 0<u \rightarrow \infty .
$$

Then there exists a complex number $A$ such that for all $u>0, q(u)=A q^{*}(u)$, where $q^{*}$ is given by Definition 1 with $\beta=-1$, i.e.

$$
q(u)=A \int_{0}^{\infty} \exp \left\{-u x+\sum_{j=1}^{m} \alpha_{j} \int_{0}^{x} \frac{1-e^{-v_{j} t}}{t} d t\right\} d x, \quad u>0 .
$$

Proof. Partial summation enables us to rewrite the differential difference equation (1.1) in the form

$$
(u q(u))^{\prime}=\sum_{j=1}^{m} c_{j}\left\{q\left(u+v_{j}\right)-q\left(u+v_{j-1}\right)\right\}, \quad u>0,
$$


where

$$
c_{j}=\sum_{i=j}^{m} \alpha_{i}, \quad j=1,2, \ldots, m .
$$

Now integrate. There exists a complex number $A$ such that for all $u>0$,

$$
u q(u)=A+\sum_{j=1}^{m} c_{j} \int_{v_{j-1}}^{v_{j}} q(u+t) d t .
$$

Let

$$
M:=\frac{4}{\lambda} \sum_{j=1}^{m}\left|c_{j}\right| e^{\lambda v_{j}} .
$$

The exponential growth requirement implies that there exist $u_{0} \geq M$ and $B>0$ such that for all $u \geq u_{0},|q(u)| \leq B e^{\lambda u}$. Inserting this inequality into (4.3) yields

$$
|u q(u)| \leq|A|+B \sum_{j=1}^{m}\left|c_{j}\right| \int_{v_{j-1}}^{v_{j}} e^{\lambda(u+t)} d t, \quad u \geq u_{0} .
$$

It follows that

$$
|q(u)| \leq \frac{|A|}{u}+\frac{B e^{\lambda u}}{\lambda u} \sum_{j=1}^{m}\left|c_{j}\right| e^{\lambda v_{j}} \leq \frac{|A|}{u}+\frac{B e^{\lambda u}}{4}, \quad u \geq u_{0} .
$$

Now we claim that $|q(x)| \leq 2|A| / x$ for $x \geq u_{0}$. To prove the claim, fix $x \geq u_{0}$ and observe that if $B e^{\lambda x} / 4 \leq|A| / x$, then (4.4) gives $|q(x)| \leq 2|A| / x$. On the other hand, if $B e^{\lambda x} / 4>|A| / x$, then we must have $B e^{\lambda u} / 4>|A| / u$ for all $u \geq x$. Since (4.4) holds for all $u \geq u_{0}$, we then get

$$
|q(u)| \leq \frac{B e^{\lambda u}}{2}, \quad u \geq x .
$$

We can now insert this latter inequality back into (4.3) and repeat the previous reasoning with $B$ replaced by $B / 2$. In general, if $n$ is a positive integer such that

$$
\frac{B e^{\lambda x}}{4 \cdot 2^{n-1}}>\frac{|A|}{x}
$$

then iterating the previous argument $n$ times will yield

$$
|q(u)| \leq \frac{|A|}{u}+\frac{B e^{\lambda u}}{4 \cdot 2^{n}}, \quad u \geq x .
$$

Note that the bound (4.5) is valid for $u \geq x$, where $x$ is the same as above. If $A \neq 0$, let $n$ be the least positive integer such that

$$
\frac{B e^{\lambda x}}{4 \cdot 2^{n}} \leq \frac{|A|}{x} .
$$

Then (4.5) gives

$$
|q(x)| \leq \frac{2|A|}{x}
$$

Since $x \geq u_{0}$ is arbitrary, (4.6) must hold for all $x \geq u_{0}$. On the other hand, if $A=0$, then letting $n \rightarrow \infty$ in (4.5) shows that $q(u)=0$ for all $u \geq x$. But again, since $x \geq u_{0}$ is arbitrary, we must have $q(u)=0$ for all $u \geq u_{0}$. In other words, (4.6) also holds for all $x \geq u_{0}$ when $A=0$. This establishes the claim. Invoking Theorem 3 now completes the proof. 
We can now show that any solution to (4.1) with $\kappa>0$ must be wildly oscillatory.

Theorem 5. Suppose that $q$ is a solution to the difference differential equation (4.1) with $\kappa>0$. Suppose further that $q$ is not a constant multiple of $q^{*}$, where $q^{*}(u)$ is given by (4.2). Then $q(u) \neq \mathrm{O}(\exp (\lambda u))$ for any fixed $\lambda>0$, and for all $u>0, q$ changes sign infinitely often in $[u, \infty)$.

Proof. Note that $\beta=-1$ in (4.1). The conclusion $q(u) \neq \mathrm{O}(\exp (\lambda u))$ for all $\lambda>0$ is an immediate consequence of Lemma 4 In particular there exists a strictly increasing sequence of positive real numbers $u_{1}<u_{2}<\ldots$ satisfying $\lim _{n \rightarrow \infty} u_{n}=\infty$ and $\left|q\left(u_{n}\right)\right|>\exp \left(u_{n}\right)$ for all positive integers $n$. Integrating the difference differential equation (4.1) yields

$$
u q(u)=A-\kappa \int_{u}^{u+1} q(t) d t, \quad u>0,
$$

where $A$ is a constant of integration which may depend on $\kappa$, but not on $u$. There is a positive integer $N$ such that if $n>N$, then $u_{n} \geq 1$ and $\exp \left(u_{n}\right)>|A|$. Let $n>N$ and set $u=u_{n}$. Suppose that $q(u)>0$. Then (4.7) yields

$$
\kappa \int_{u}^{u+1} q(t) d t=A-u q(u)<|A|-e^{u}<0 .
$$

Since $\kappa>0$, it follows that

$$
\int_{u}^{u+1} q(t) d t<0
$$

and hence $q$ must change sign from positive to negative somewhere in the half-open, half-closed interval $(u, u+1]$. If, on the other hand, $q(u)<0$, then (4.7) yields

$$
\kappa \int_{u}^{u+1} q(t) d t=A-u q(u)>-|A|+e^{u}>0,
$$

and hence $q$ must change sign from negative to positive somewhere in $(u, u+1]$. Thus, we have shown that $q$ has a sign change beyond $u_{n}$ for each $n>N$, and the proof is complete.

\section{The ADJOINT RELATION}

We would like to remove the restriction $\beta=-1$ in Lemma 4 A careful examination of the proof of Lemma 4 suggests that the iterative argument employed therein succeeded because the lengths of the ranges of integration in the representation (4.3) are all independent of $u$. Note that (4.3) is equivalent to

$$
u q(u)=A+\sum_{j=1}^{m} \alpha_{j} \int_{u-v_{j}}^{u} q\left(t+v_{j}\right) d t .
$$

In order to recover this property in the case when $\beta \neq-1$, let us seek a function $p$ such that for some constant $A$, the equation

$$
u p(u) q(u)=A+\sum_{j=1}^{m} \alpha_{j} \int_{u-v_{j}}^{u} p(t) q\left(t+v_{j}\right) d t
$$


holds for all sufficiently large values of $u$. Let us suppose further that beyond some point, $p$ is differentiable. Differentiating (5.1) with respect to $u$ reveals that

$$
\begin{aligned}
& u q^{\prime}(u) p(u)+u p^{\prime}(u) q(u)+p(u) q(u) \\
& \quad=p(u) \sum_{j=1}^{m} \alpha_{j} q\left(u+v_{j}\right)-q(u) \sum_{j=1}^{m} \alpha_{j} p\left(u-v_{j}\right) .
\end{aligned}
$$

If we now substitute the right-hand side of the difference differential equation (1.1) for $u q^{\prime}(u)$ in this latter equation, the first sum on the right-hand side can be cancelled with the corresponding sum on the left. The result is

$$
\alpha_{0} p(u) q(u)+u p^{\prime}(u) q(u)+p(u) q(u)=-q(u) \sum_{j=1}^{m} \alpha_{j} p\left(u-v_{j}\right),
$$

which is certainly the case if $p$ satisfies the delay differential equation (1.2). Conversely, it is clear that if $q$ satisfies (1.1) and $p$ satisfies (1.2), then (5.1) must also hold for all sufficiently large values of $u$.

\section{The FUnCtion $u \mapsto p(u, a, b)$}

Our plan is to use the adjoint equation (5.1) to deduce the asymptotic behavior of $q(u)$ for large $u$ using only a very weak estimate on the growth rate of $q(u)$. In order to do this, we need reasonably precise knowledge concerning the rate of growth of a non-trivial solution $p(u)$ to the delay differential equation (1.2) as $u$ increases without bound. Most of what we need can be found in [35], where the existence of an asymptotic expansion for $p$ for the case (in our notation) $\Re\left(\alpha_{0}\right)<0$ is proved, and the first term of the expansion is determined explicitly. Nevertheless, it is worthwhile to obtain some additional results, and for this it is useful to extend Wheeler's 41, 42] more comprehensive treatment of the $m=2$ case to arbitrary $m$ and complex $\alpha_{j}$. Therefore, with regard to the choice of boundary condition and the location and classification of discontinuities, our development in this section more closely parallels that of [42. However, to provide an explicit formula for all coefficients in the asymptotic expansion, we find it more convenient to extend the technique used in 35. To facilitate comparison with our results, we set $a=1+\alpha_{0}$ and let $b$ denote the vector $\left(\alpha_{1}, \alpha_{2}, \ldots, \alpha_{m}\right)$.

Let

$$
C_{0}=\prod_{j=1}^{m}\left(v_{j} e^{\gamma}\right)^{-\alpha_{j}}
$$

where, as usual,

$$
\gamma=\lim _{n \rightarrow \infty}\left(\sum_{j=1}^{n} \frac{1}{j}-\log n\right)
$$

is Euler's constant. Consider a particular solution $p(u)=p(u, a, b)$ of the delay differential equation (1.2), which is defined uniquely for all real $u$ by the following 
six conditions:

$$
\begin{aligned}
& (u p(u))^{\prime}=-\sum_{j=0}^{m} \alpha_{j} p\left(u-v_{j}\right) ; \\
& p(u)=0 \text { whenever } u \leq 0 ; \\
& p(u)=\frac{C_{0}}{\Gamma(1-a)} u^{-a} \text { if } 0<u \leq v_{1} ;
\end{aligned}
$$

$p(u)$ is continuous for $u>0$ when $\Re(a)<1$;

$p(u, a+1, b)=\frac{d}{d u} p(u, a, b) ;$

$p$ is continuous from the left at every point.

We need to prove that such a unique function exists. First assume $\Re(a)<1$. The function defined by (6.1c) is integrable for $0<u \leq v_{1}$ and the delay differential equation (6.1a) can be rewritten as

$$
\left(u^{a} p(u)\right)^{\prime}=-u^{a-1} \sum_{j=1}^{m} \alpha_{j} p\left(u-v_{j}\right),
$$

which can be integrated forward on successive intervals. That is, for each positive integer $n$, if $n<u \leq n+1$, then

$$
p\left(u v_{1}\right)=u^{-a}\left\{n^{a} p\left(n v_{1}\right)-\int_{n}^{u} t^{a-1} \sum_{j=1}^{m} \alpha_{j} p\left(t v_{1}-v_{j}\right) d t\right\} .
$$

This establishes uniqueness and every condition except (6.1e). To see that 6.1e) holds, simply differentiate (6.1a) and (6.1c) and apply the functional equation for the gamma function. For the case $\Re(a) \geq 1$, we use the fact that if $n$ is a positive integer, then

$$
p(u, a, b)=\left(\frac{d}{d u}\right)^{n} p(u, a-n, b),
$$

which follows from (6.1e).

From (6.2), it follows that $p$ has a two-sided derivative at every point $x$ for which $p$ is continuous at each of the points $x-v_{j}, 1 \leq j \leq m$. Otherwise, we need to take derivatives from the left in (6.1a) and (6.1e). This we can do, again by (6.2), since $p$ is continuous from the left at every point.

Theorem 6. (i) If $\Re(a)$ is not an integer, then the set of points at which $p$ is discontinuous is precisely $\left\{n v_{j}: n \in \mathbf{Z}, 0 \leq n<\Re(a), 1 \leq j \leq m\right\}$. At these points, $p$ has a finite limit from the left, and is unbounded from the right. Moreover, if $n$ is a non-negative integer, $\Re(a)>n$, and $1 \leq j \leq m$, then

$$
p(u) \sim \frac{\left(-\alpha_{j}\right)^{n} C_{0}}{n ! v_{j}^{n} \Gamma(n-a+1)}\left(u-n v_{j}\right)^{n-a}, \quad u \rightarrow n v_{j}+,
$$

whereas $\lim _{u \rightarrow n v_{j}-} p(u)=p\left(n v_{j}\right)$ is finite. 
(ii) If $\Re(a)$ is a non-negative integer, but a is not itself an integer, then the set of points at which $p$ is discontinuous is precisely $\left\{n v_{j}: n \in \mathbf{Z}, 0 \leq n \leq \Re(a), 1 \leq\right.$ $j \leq m\}$. The discontinuities consist of two types. If $n$ is a non-negative integer, $\Re(a)>n$, and $1 \leq j \leq m$, then as in (i),

$$
p(u) \sim \frac{\left(-\alpha_{j}\right)^{n} C_{0}}{n ! v_{j}^{n} \Gamma(n-a+1)}\left(u-n v_{j}\right)^{n-a}, \quad u \rightarrow n v_{j}+,
$$

whereas $\lim _{u \rightarrow n v_{j}-} p(u)=p\left(n v_{j}\right)$ is finite. If $n=\Re(a)$, then $p$ is bounded in a neighbourhood of $n v_{j}$, but $\lim _{u \rightarrow n v_{j}+} p(u)$ does not exist.

(iii) If a is a positive integer, then $p$ has only finite jump discontinuities. These can only occur at the points $n v_{j}$, where $n$ is an integer, $1 \leq n \leq a$, and $1 \leq j \leq m$.

(iv) Finally, $p(u, 0, b)$ has a finite jump discontinuity at $u=0$ and no other discontinuities.

Sketch of the proof. The statements of the theorem are true if $\Re(a)<1$ as we have already noted. The other cases can be established by induction, using the equation

$$
p(u, a+1, b)=\frac{d}{d u} p(u, a, b)=-\frac{a}{u} p(u, a, b)-\frac{1}{u} \sum_{j=1}^{m} \alpha_{j} p\left(u-v_{j}, a, b\right),
$$

which is certainly valid if $u$ is not an integer multiple of $v_{j}, 1 \leq j \leq m$. For details, see [42] Theorem 1].

Remark. The situation in (iii) is somewhat unsatisfactory, as in general all we can say is that if $a$ is a positive integer and $u$ is a point of discontinuity, then necessarily $u=n v_{j}$ for some positive integer $n \leq a$ and $1 \leq j \leq m$. Although it is typically the case that all such points are indeed points of discontinuity-for example, this is indeed true if the $m$ positive real numbers $v_{1}, \ldots, v_{m}$ are linearly independent over the integers - it may happen that for certain values of the parameters $\alpha_{j}, v_{j}$, the jumps arising from different terms in (6.3) cancel each other. As an example, consider the case $m=2, a=2, b=\left(\alpha_{1}, \alpha_{2}\right)=(1,-2), v_{1}=1, v_{2}=2$. Then $C_{0}=4 e^{\gamma}$. We find that the jump at $2 v_{1}+$ is exactly cancelled by the jump at $v_{2}+$, so that $p(2+, 2, b)=e^{\gamma}=p(2-, 2, b)$. As noted by Wheeler [42, such cancellations cannot occur when $m=1$.

We now turn to the problem of determining the behavior of $p(u)$ for large positive real $u$. We take the following result of Levin and Fainleib 35] as our point of departure, recasting it in our notation, and with our boundary condition (6.1c), etc.

Lemma 7 ([35, Lemma 1.3.1). Let $\Re(a)<1$, and let $\varphi(u)=(u \log u) / v_{m}+\mathrm{O}(u)$, $0<u \rightarrow \infty$. We have the following behavior for $p(u)$ as $0<u \rightarrow \infty$ :

(i) If $\beta$ is a non-negative integer, then $p(u)=\mathrm{O}(\exp (-\varphi(u)))$.

(ii) If $\beta$ is a negative integer, there exists a polynomial $r_{\beta}$ of degree $-\beta-1$ such that $p(u)=r_{\beta}(u)+\mathrm{O}(\exp (-\varphi(u)))$.

(iii) For any complex $\beta$ not an integer, there is an asymptotic expansion $p(u) \sim$ $\sum_{n \geq 0} c_{n} u^{-\beta-1-n}$.

Levin and Fainleib [35] did not identify the polynomial $r_{\beta}$ in (ii). In addition, they gave only the first term in the asymptotic expansion (iii). We shall determine all the coefficients $c_{n}$ and the polynomial $r_{\beta}$ explicitly. We shall also remove the 
restriction on $\Re(a)$. To carry this out, we need the following formula for the Laplace transform of $p$.

Theorem 8. Let $\Re(a)<1$ and $\Re(s)>0$. Then

$$
\int_{0}^{\infty} e^{-s u} p(u) d u=s^{\beta} \exp \left\{-\sum_{j=1}^{m} \alpha_{j} \int_{0}^{s} \frac{1-e^{-t v_{j}}}{t} d t\right\} .
$$

If $\beta$ is a non-negative integer, the formula is valid for all complex numbers $s$.

Theorem 8 is actually just a restatement in our notation of a corresponding result derived in 35. Although the region of convergence was not discussed there, both the existence of the Laplace transform and its region of convergence follow easily from the growth behavior of $p$ as given in Lemma 7

Let $n$ be a non-negative integer. Recall the polynomial [9] equation (3.2)]

$$
Q_{n}(u, b)=\left.\left(\frac{\partial}{\partial z}\right)^{n}\right|_{z=0} \exp \left\{u z-\sum_{j=1}^{m} \alpha_{j} \int_{0}^{z} \frac{e^{v_{j} t}-1}{t} d t\right\} .
$$

Remembering that $b$ denotes the vector of coefficients $\left(\alpha_{1}, \ldots, \alpha_{m}\right)$, we let $Q_{n}(u,-b)$ be the polynomial obtained by negating each $\alpha_{j}(1 \leq j \leq m)$ in $Q_{n}(u, b)$. With this notation, we can now restate Lemma 7 with the coefficients $c_{n}$ and the polynomial $r_{\beta}$ explicitly identified.

Theorem 9. Let $\varphi(u)$ be as in the statement of Lemma 7. As $0<u \rightarrow \infty$, we have the following behavior for $p(u)$ :

(i) If $\beta$ is a non-negative integer, then $p(u)=\mathrm{O}(\exp (-\varphi(u)))$.

(ii) If $\beta$ is a negative integer, then

$$
p(u)=\sum_{n=0}^{-\beta-1} \frac{(-1)^{n}}{n !} \frac{Q_{n}(0,-b)}{(-\beta-1-n) !} u^{-\beta-1-n}+\mathrm{O}\left(e^{-\varphi(u)}\right) .
$$

(iii) For any complex number $\beta$ not an integer, we have the asymptotic expansion

$$
p(u) \sim \sum_{n=0}^{\infty} \frac{(-1)^{n}}{n !} \frac{Q_{n}(0,-b)}{\Gamma(-\beta-n)} u^{-\beta-1-n} .
$$

Proof. In view of Lemma 7 there is nothing to prove for (i), and the error term need not concern us in (ii). Fix a positive integer $h$. It is sufficient to consider the case $\Re(a)<1$ and $\Re(\beta)<1-h$, since as noted in [35], the remaining cases reduce to the problem of differentiating asymptotic expansions, and by (6.1a) this is legitimate. Let $0<\varepsilon<1$ be such that $-\Re(\beta)-h-\varepsilon>-1$. Substituting

$$
p(u)=\sum_{n=0}^{h-1} c_{n} u^{-\beta-1-n}+\mathrm{O}\left(u^{-\Re(\beta)-h-\varepsilon}\right), \quad 0<u \rightarrow \infty,
$$

into the Laplace transform for $p$, we find that

$$
\int_{0}^{\infty} e^{-s u} p(u) d u=\sum_{n=0}^{h-1} c_{n} \Gamma(-\beta-n) s^{\beta+n}+\mathrm{O}\left(s^{\Re(\beta)+h+\varepsilon-1}\right), \quad s \rightarrow 0+.
$$


On the other hand, Theorem 8 and the definition of the polynomials $Q_{n}(0,-b)$ imply that

$$
\int_{0}^{\infty} e^{-s u} p(u) d u \sim \sum_{n=0}^{\infty} \frac{(-1)^{n}}{n !} Q_{n}(0,-b) s^{\beta+n}, \quad s \rightarrow 0+.
$$

Comparing the two expressions for the Laplace transform, we infer that

$$
c_{n}=\frac{(-1)^{n}}{n !} \frac{Q_{n}(0,-b)}{\Gamma(-\beta-n)},
$$

which proves (iii). If $\beta$ is an integer and $\beta \geq-n$, we see that $c_{n}=0$. It follows that if $\beta$ is a negative integer, then

$$
r_{\beta}(u)=\sum_{n=0}^{-\beta-1} \frac{(-1)^{n}}{n !} \frac{Q_{n}(0,-b)}{\Gamma(-\beta-n)} u^{-\beta-1-n},
$$

as stated in (ii).

\section{ExPONENTIALLY BOUNDED SOLUTIONS}

In this section we prove that, up to a multiplicative constant, the difference differential equation (1.1) has a unique solution in the class of functions majorized by a function of exponential growth. We first establish an inequality for certain exponential integrals that arise in the proof.

Lemma 10. Let $u, r$ and $\lambda$ be real numbers satisfying $\lambda>r^{+}=\max (r, 0)$ and $u>1$. Then

$$
\int_{1}^{u} e^{\lambda t} t^{-r} d t \leq \frac{e^{\lambda u} u^{-r}}{\lambda-r^{+}}
$$

Proof. Denote the integral by $I$. An easy integration by parts shows that

$$
I \leq \frac{1}{\lambda} e^{\lambda u} u^{-r}+\frac{r}{\lambda} \int_{1}^{u} e^{\lambda t} t^{-r-1} d t .
$$

If $r<0$, then the coefficient of the integral on the right-hand side of (7.1) is negative, and so the corresponding term can be dropped, yielding

$$
I \leq \frac{1}{\lambda} e^{\lambda u} u^{-r} .
$$

Since $r<0$ implies $r^{+}=0$, the claim is established in this case. On the other hand, if $r \geq 0$, then $t^{-r} \geq t^{-r-1}$ for $1 \leq t \leq u$ and hence

$$
I \leq \frac{1}{\lambda} e^{\lambda u} u^{-r}+\frac{r}{\lambda} \int_{1}^{u} e^{\lambda t} t^{-r} d t=\frac{1}{\lambda} e^{\lambda u} u^{-r}+\frac{r}{\lambda} I .
$$

Since $\lambda>r \geq 0$, it follows that

$$
I \leq \frac{e^{\lambda u} u^{-r}}{\lambda-r}
$$

Since $r \geq 0$ implies $r^{+}=r$, the claim is established in this case also. 
Theorem 11. Suppose that $q$ satisfies the difference differential equation (1.1). Suppose further that there exists a positive real number $\lambda$ such that

$$
q(u)=\mathrm{O}\left(e^{\lambda u}\right), \quad 0<u \rightarrow \infty .
$$

Then there exists a complex number $A$ such that for all $u>0, q(u)=A q^{*}(u)$, where $q^{*}$ is given by Definition 1 . The multiplicative constant A may of course depend on the parameters $\alpha_{j}, v_{j}(j=0,1,2, \ldots, m)$ but not on $u$.

Proof. First, suppose that $\beta=n$ is a non-negative integer. The difference differential equation (1.1) implies that $q^{\prime}(u)$ satisfies (1.1) with $\alpha_{0}$ replaced by $\alpha_{0}-1$, and that $q^{\prime}(u)=\mathrm{O}\left(u^{-1} e^{\lambda u}\right), 0<u \rightarrow \infty$. Hence, we deduce that the $n+1^{\text {st }}$ derivative of $q$ satisfies the conditions of Lemma 4 . Accordingly, by Lemma 4 and Proposition 1, there exists a complex constant $A$ such that

$$
q^{(n+1)}(u) \sim A u^{-1}, \quad 0<u \rightarrow \infty .
$$

It follows that

$$
q^{(n)}(u) \sim A \log u, \quad 0<u \rightarrow \infty .
$$

Also, $q^{(n)}$ satisfies the difference differential equation (1.1) with $\beta=0$. Theorem 3 now implies that $q^{(n)}$ is a constant multiple of $q^{*}$, where $q^{*}$ is given by Definition 1 with $\beta=0$. It follows that $A=0$ and $q^{(n)}$ is constant. Hence, there exist complex numbers $a_{0}, a_{1}, \ldots, a_{n}$ such that for all $u>0$,

$$
q(u)=\sum_{j=0}^{n} a_{j} u^{j}
$$

More specifically, Theorem 2 implies that $q=a_{n} Q_{n}$, where

$$
Q_{n}(u):=\left.\left(\frac{\partial}{\partial z}\right)^{n}\right|_{z=0} \exp \left\{u z-\sum_{j=0}^{m} \alpha_{j} \int_{0}^{z} \frac{e^{v_{j} t}-1}{t} d t\right\}
$$

is the polynomial obtained by setting $\beta=n$ in $(3.1 \mathrm{~b})$ of Definition 1 and deforming the contour into the unit circle. See [9] for additional properties of the polynomial $Q_{n}$.

For the general case, we assume that $\beta$ is a complex number, but not a nonnegative integer. Let $c=\Re(\beta)$. Since increasing $\lambda$ only weakens the hypothesis, we may assume that $K:=\lambda-\max (c+1,0)>0$. Let

$$
M:=\frac{16}{K} \sum_{j=1}^{m}\left|\alpha_{j}\right| e^{\lambda v_{j}} .
$$

By hypothesis, there exist $u_{1} \geq M$ and $B>0$ such that for all $u \geq u_{1},|q(u)| \leq$ $B e^{\lambda u}$. Let $p(u)=\Gamma(-\beta) p(u, a, b)$, where $p(u, a, b)$ is as in 6 . Since $Q_{0}(0,-b)=1$, Theorem 9 implies that $p(u) \sim u^{-\beta-1}$ as $0<u \rightarrow \infty$. Therefore, there exists a positive real number $u_{2}$ such that for all $u \geq u_{2}, \frac{1}{2} u^{-c-1} \leq|p(u)| \leq 2 u^{-c-1}$. There must also exist a complex number $A$ and a positive real number $u_{3}$ such that the adjoint relation (cf.5.1)

$$
u p(u) q(u)=A+\sum_{j=1}^{m} \alpha_{j} \int_{u-v_{j}}^{u} p(t) q\left(t+v_{j}\right) d t
$$


holds for all $u \geq u_{3}$. Let $u_{4}:=\max \left(u_{1}, u_{2}+v_{m}, u_{3}, 1+v_{m}\right)$. Then for all $u \geq u_{4}$, by Lemma 10 we have

$$
\begin{aligned}
|u p(u) q(u)| & \leq|A|+2 B \sum_{j=1}^{m}\left|\alpha_{j}\right| \int_{u-v_{j}}^{u} t^{-c-1} e^{\lambda\left(t+v_{j}\right)} d t \\
& \leq|A|+2 B K^{-1} u^{-c-1} \sum_{j=1}^{m}\left|\alpha_{j}\right| e^{\lambda\left(u+v_{j}\right)} \\
& \leq|A|+\frac{u_{1}}{8} B u^{-c-1} e^{\lambda u} .
\end{aligned}
$$

Let $A^{\prime}=2 A$. It follows that

$$
|q(u)| \leq\left|A^{\prime} u^{\beta}\right|+\frac{u_{1}}{4 u} B e^{\lambda u} \leq\left|A^{\prime} u^{\beta}\right|+\frac{1}{4} B e^{\lambda u}, \quad u \geq u_{4} .
$$

Now let $u_{0}:=\max \left(u_{4}, c / \lambda\right)$, and fix $x \geq u_{0}$. If $\frac{1}{4} B e^{\lambda x} \leq\left|A^{\prime} x^{\beta}\right|$, then (7.3) gives $|q(x)| \leq 2\left|A^{\prime} x^{\beta}\right|$.

On the other hand, if $\frac{1}{4} B e^{\lambda x}>\left|A^{\prime} x^{\beta}\right|$, then we claim that $\frac{1}{4} B e^{\lambda u}>\left|A^{\prime} u^{\beta}\right|$ for all $u \geq x$. To see the claim, let $f(u):=\log B-\log 4+\lambda u-\log \left|A^{\prime}\right|-\Re(\beta) \log u$. We have $f(x)>0$ and $f^{\prime}(u)=\lambda-c / u>0$ for all $u \geq x$. Therefore, $f$ is positive and strictly increasing on the interval $[x, \infty)$, and this proves the claim. Thus, in this case, (7.3) gives $|q(u)| \leq \frac{1}{2} B e^{\lambda u}$ for all $u \geq x$. Now insert this latter inequality back into the adjoint relation (7.2), i.e. repeat the previous argument with $B$ replaced by $B / 2$. We get

$$
|q(u)| \leq\left|A^{\prime} u^{\beta}\right|+\frac{1}{8} B e^{\lambda u}, \quad u \geq x .
$$

In general, if $n$ is a positive integer such that

$$
\frac{B e^{\lambda x}}{4 \cdot 2^{n-1}}>\left|A^{\prime} x^{\beta}\right|
$$

then $n$ iterations of the preceding argument will yield

$$
|q(u)| \leq\left|A^{\prime} u^{\beta}\right|+\frac{B e^{\lambda u}}{4 \cdot 2^{n}}, \quad u \geq x .
$$

Therefore, if $A^{\prime} \neq 0$, let $n$ be the least positive integer such that

$$
\frac{B e^{\lambda x}}{4 \cdot 2^{n}} \leq\left|A^{\prime} x^{\beta}\right|
$$

Then (7.5) implies that

$$
|q(x)| \leq 2\left|A^{\prime} x^{\beta}\right| .
$$

Since $x \geq u_{0}$ is arbitrary, the bound (7.6) must hold for all such $x$. On the other hand, if $A^{\prime}=0$, then letting $n \rightarrow \infty$ in (7.5) shows that $q(u)=0$ for all $u \geq x$. But again $x \geq u_{0}$ is arbitrary, so we must have $q(u)=0$ for all $u \geq u_{0}$. In other words, the bound (7.6) holds for all $x \geq u_{0}$ and all complex numbers $A^{\prime}$. Invoking Theorem 3 completes the proof. 


\section{FinAL REMARKS}

The introduction of an adjoint equation as an aid to studying solutions of difference differential equations goes back at least to de Bruijn's study of the Buchstab function [10]. There and subsequently (see eg. [36, 15, 41, 42]), the adjoint equation is used to deduce information about a solution to a special case of (1.2) from a solution to the corresponding special case of 1.1). In this paper, we turned the process around, deducing information about a solution to (1.1) from the adjoint equation and a solution to (1.2).

It is interesting to compare and contrast the behavior of $p$ and $q$ near zero and infinity. As in $\$ 7$ let $p(u)=\Gamma(-\beta) p(u, a, b)$, where $p(u, a, b)$ is as in $\$ 6$ Assume that $\beta$ is not a non-negative integer. Then Theorem 9 implies that

$$
p(u) \sim \sum_{n=0}^{\infty}(-1)^{n}\left(\begin{array}{c}
-\beta-1 \\
n
\end{array}\right) Q_{n}(0,-b) u^{-\beta-1-n}, \quad 0<u \rightarrow \infty,
$$

whereas Theorem 5 of [9] states that

$$
q(u) \sim \sum_{n=0}^{\infty}\left(\begin{array}{l}
\beta \\
n
\end{array}\right) Q_{n}(0, b) u^{\beta-n}, \quad 0<u \rightarrow \infty .
$$

If in addition, $\Re\left(\alpha_{0}\right)<0$, then Theorem $7($ ii) of [9] can be restated as

$$
q(u) \sim u^{\alpha_{0}} \frac{\Gamma\left(-\alpha_{0}\right)}{\Gamma(-\beta)} \prod_{j=1}^{m}\left(v_{j} e^{\gamma}\right)^{\alpha_{j}}, \quad u \rightarrow 0+,
$$

whereas (6.1c) implies that

$$
p(u)=u^{-\alpha_{0}-1} \frac{\Gamma(-\beta)}{\Gamma\left(-\alpha_{0}\right)} \prod_{j=1}^{m}\left(v_{j} e^{\gamma}\right)^{-\alpha_{j}}, \quad 0<u \leq v_{1} .
$$

In particular, $\lim _{u \rightarrow 0+} u p(u) q(u)=\lim _{u \rightarrow+\infty} u p(u) q(u)=1$.

\section{ACKNOWLEDGMent}

It is a pleasure to thank the referee for a careful examination of the paper, and for several insightful suggestions which led to improvements in the exposition.

\section{REFERENCES}

[1] R. Arratia, A. D. Barbour and S. Tavaré, "Random Combinatorial Structures and Prime Factorizations," Notices Amer. Math. Soc. 44 (1997), no. 8, 903-910. MR 98i:60007

[2] R. Arratia, and S. Tavaré, "The cycle structure of random permutations," Ann. Probab. 20 (1992), 1567-1591. MR 93g:60013

[3] K. Alladi, "An Erdös-Kac theorem for integers without large prime factors," Acta Arithmetica 49 (1987), 81-105. MR 89b:11077

[4] N. C. Ankeny and H. Onishi, "The general sieve," Acta Arithmetica 10 (1964), 31-62. MR 29:4740

[5] V. Balakrishnan, G. Sankaranarayanan and C. Suyambulingom, "Ordered cycle lengths in a random permutation," Pacific J. Math. 36 (1971), 603-613. MR 52:12037

[6] P. Billingsley, "On the distribution of large prime divisors," Periodica Mathematica Hungarica 2 (1972), 283-289. MR 49:243

[7] D. Bradley, "A sieve auxiliary function," Ph.D. Thesis, University of Illinois, Urbana, 1995.

[8] D. Bradley, "A sieve auxiliary function," in Analytic Number Theory: Proceedings of a Conference in Honor of Heini Halberstam (B. Berndt et al. eds.), Progress in Math. 138, Birkhäuser, Boston (1996), 173-210. MR 97h:11099 
[9] D. Bradley and H. Diamond, "A difference differential equation of Euler-Cauchy type," J. Differential Equations 138 (1997), no. 2, 267-300. MR 99a:34172

[10] N. G. de Bruijn, "On the number of uncancelled elements in the sieve of Eratosthenes," Nederl. Akad. Wetensch. Proc. (6) 53 (1950), 803-821. MR 12:11d

[11] N. G. de Bruijn, "On the number of positive integers $\leq x$ and free of prime factors $>y$," Nederl. Akad. Wetensch. Proc. Ser. A 54 (Indag. Math. 13) (1951), 50-60. MR 13:724e

[12] N. G. de Bruijn, "On the number of positive integers $\leq x$ and free of prime factors $>y$ II," Indag. Math. 28 (1966), 239-247. MR 34:5770

[13] N. G. de Bruijn and J. H. van Lint, "Incomplete sums of multiplicative functions I, II," Nederl. Akad. Wetensch. Proc. Ser. A 67 (Indag. Math. 26) (1964), 339-347; 348-359. MR 30:4731

[14] A. A. Buchstab, "Asymptotic estimates of a general number-theoretic function," Mat. Sbornik 44 (1937), 1239-1246.

[15] A. Y. Cheer and D. A. Goldston, "A differential delay equation arising from the sieve of Eratosthenes," Math. Comp. 55 (1990), 129-141. MR 90j:11091

[16] H. Diamond, H. Halberstam, and H.-E. Richert, "Combinatorial sieves of dimension exceeding one," J. Number Theory 28 (1988), 306-346. MR 89g:11080

[17] H. Diamond, H. Halberstam, and H.-E. Richert, "Combinatorial sieves of dimension exceeding one II," in Analytic Number Theory: Proceedings of a Conference in Honor of Heini Halberstam (Bruce C. Berndt et al. eds.), Progress in Math. 138, Birkhäuser, Boston (1996), 265-308. MR 97e:11112

[18] H. Diamond, H. Halberstam, and H.-E. Richert, "A boundary value problem for a pair of differential delay equations related to sieve theory I," in Analytic Number Theory: Proceedings of a Conference in Honor of P. Bateman (B. Berndt et al. eds.), Birkhäuser, Boston (1990), 133-157. MR 92a:11107.

[19] H. Diamond, H. Halberstam, and H.-E. Richert, "A boundary value problem for a pair of differential delay equations related to sieve theory II," J. Number Theory 45 (1993), 129-185. MR 94j:11089

[20] H. Diamond, H. Halberstam, and H.-E. Richert, "A boundary value problem for a pair of differential delay equations related to sieve theory III," J. Number Theory 47 (1994), 300-328. MR 95e:11100

[21] H. Diamond, H. Halberstam, and H.-E. Richert, "Sieve auxiliary functions," in Number Theory: Proceedings of the First Conference of the Canadian Number Theory Association (Richard Mollin ed.), W. de Gruyter \& Co. (1990), 99-113. MR 92f:11122

[22] H. Diamond, H. Halberstam, and H.-E. Richert, "Sieve auxiliary functions II," in A tribute to Emil Grosswald: Number Theory and Related Analysis (Marvin Knopp et al. eds.) Contemporary Math., 143 Amer. Math. Soc., Providence, RI (1993), 247-253. MR 94c:11090

[23] H. Diamond, H. Halberstam, and H.-E. Richert, "Estimation of the sieve auxiliary functions $q_{\kappa}$ in the range $1<\kappa<2, "$ Analysis 14 (1994), 75-102. MR 95g:11091

[24] K. Dickman, "On the frequency of numbers containing primes of a certain relative magnitude," Ark. Mat. Ast. Fys. 22 (1930), 1-14.

[25] S. W. Golomb, "Random permutations," Bull. Amer. Math. Soc. 70 (1964), 747.

[26] V. Goncharov, "Sur la distribution des cycles dans les permutations," C. R. (Doklady) Acad. Sci. URSS 35 (1942), 267-269. MR 4:102g

[27] F. Grupp, "On difference-differential equations in the theory of sieves," J. Number Theory 24 (1986), 154-173. MR 87k:11101

[28] H. Halberstam and H.-E. Richert, Sieve Methods, Academic Press, London, 1974. MR 54:12689

[29] D. Hensley, "The sum of $\alpha^{\Omega(n)}$ over integers $n \leq x$ with all factors between $\alpha$ and $y$," $J$. Number Theory 18 (1984), 206-212. MR 85i:11071

[30] A. Hildebrand, "On the number of positive integers $\leq x$ and free of prime factors $>y$, $J$. Number Theory 22 (1986), 289-307. MR 87d:11066

[31] A. Hildebrand, "The asymptotic behavior of the solutions of a class of differential-difference equations," J. London Math. Soc. (2) 42 (1990), no. 1, 11-31. MR 92f:11123

[32] A. Hildebrand and G. Tenenbaum, "On a class of differential-difference equations arising in number theory," J. Anal. Math. 61 (1993), 145-179. MR 94i:11069

[33] H. Iwaniec, "Rosser's sieve," Acta Arithmetica 36 (1980), 171-202. MR 81m:10086 
[34] D. E. Knuth and L. T. Pardo, "Analysis of a simple factorization algorithm," Theor. Comp. Sci. 3 (1976), 321-348. MR 58:16485

[35] B. V. Levin and A. S. Fainleib, "Application of some integral equations to problems of number theory," Russian Math. Surveys 22 (1967), 119-204. MR 37:5174

[36] H. Maier, "Primes in short intervals," Michigan Math. J., 32 (1985), 221-225. MR 86i:11049

[37] P. Moree, "Psixyology and Diophantine Equations," Ph.D. Thesis, Rijksuniversiteit te Leiden, 1993. MR 96e:11114

[38] P. W. Purdom and J. H. Williams, "Cycle length in a random function," Trans. Amer. Math. Soc. 133 (1968), 547-551. MR 37:3616

[39] H. J. J. te Riele, "Numerical solution of two coupled nonlinear equations related to the limits of Buchstab's iteration sieve," Afdeling Numerieke Wiskunde 86, Mathematisch Centrum, Amsterdam, 1980. MR 81j:65048

[40] L. Shepp and S. P. Lloyd, "Ordered cycle lengths in a random permutation," Trans. Amer. Math. Soc. 121 (1966), 340-357. MR 33:3320

[41] F. Wheeler, "On two differential-difference equations arising in analytic number theory," Ph.D. Thesis, University of Illinois, Urbana, 1988.

[42] F. Wheeler, "Two differential-difference equations arising in number theory," Trans. Amer. Math. Soc. 318 (1990), 491-523. MR 90g:11134

Department of Mathematics and Statistics, University of Maine, 5752 Neville Hall, Orono, Maine 04469-5752

E-mail address: dbradley@member.ams.org

E-mail address: bradley@math.umaine.edu 